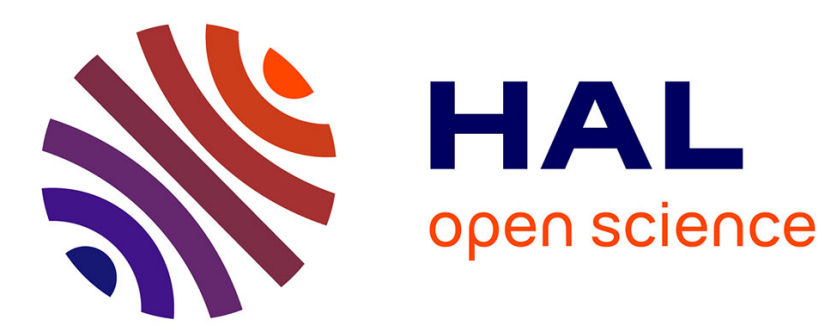

\title{
INTELLIGIBILITÉ DE LA PAROLE EN CHAMBRE SOURDE - INFLUENCE DU DIFFUSEUR
}

\author{
A. Randrianarison, C. Legros
}

\section{To cite this version:}

A. Randrianarison, C. Legros. INTELLIGIBILITÉ DE LA PAROLE EN CHAMBRE SOURDE INFLUENCE DU DIFFUSEUR. Journal de Physique IV Proceedings, 1992, 02 (C1), pp.C1-343-C1346. 10.1051/jp4:1992174. jpa-00251246

\section{HAL Id: jpa-00251246 https://hal.science/jpa-00251246}

Submitted on 1 Jan 1992

HAL is a multi-disciplinary open access archive for the deposit and dissemination of scientific research documents, whether they are published or not. The documents may come from teaching and research institutions in France or abroad, or from public or private research centers.
L'archive ouverte pluridisciplinaire HAL, est destinée au dépôt et à la diffusion de documents scientifiques de niveau recherche, publiés ou non, émanant des établissements d'enseignement et de recherche français ou étrangers, des laboratoires publics ou privés. 


\title{
INTELLIGIBILITE DE LA PAROLE EN CHAMBRE SOURDE - INFLUENCE DU DIFFUSEUR
}

\author{
A.L. RANDRIANARISON et C. LEGROS \\ Laboratoire d'Acoustique, de Métrologie et d'Instrumentation, 38 rue des 36 Ponts, F-31400 Toulouse, \\ France
}

\begin{abstract}
Résumé. - On considère une situation courante où un système de sonorisation (constitué en bout de chaîne par des enceintes acoustiques ) diffuse des messages de parole dans des locaux particulièrement bruyants. L'objectif des recherches est d'optimiser, grâce à une forme de régression, les scores d'intelligibilité en un point du local pour chaque valeur du rapport signalsur-bruit. Certains coefficients $(\mathrm{Q}$ et $\mathrm{N}$ ) associés à ce modèle mathématique sont analysés selon que l'on se trouve dans des salles réverbérantes de volumes très variables ou en chambre sourde. Dans cette dernière catégorie de local, d'autres types d'expérimentations sont menés afin d'étudier l'influence du diffuseur sur les coefficients. Il apparâit que le coefficient $Q$ dépendrait en partie de l'enceinte considérée sans qu'il soit pour autant indépendant du paramètre $N$. Les résultats présentés ici sont ceux d'une serie d'expérimentations où un bruit blanc est utilisé comme bruit de masque. Le signal de parole est constitué de listes de mots de trois phonèmes de la langue française (listes de mots phonétiquement équilibrées et non nécessairement pourvues de signification).
\end{abstract}

\begin{abstract}
The following situation is considered: a sonorisation system, ending by loudspeakers radiating speech signals in particularly noisy rooms. The aim of the study is to optimize, thanks to a mathematical regression form, the speech intelligibility, at each point, for each value of the signal-to-noise ratio. Coefficients $(Q$ and $N)$ associated to this model are investigated, according to reverberant and anechoïc chamber. In this last type of local, other experiences are driven for examining loudspeaker effect on the Q-N parameters. It appears that Qcoefficient depends on the used baffle without being uncorrelated with $N$. Results are shown for series of experiences where a white-noise is the masking-signal. The speech signals are french lists of Phonetically-Balanced, trisyllabic and not necessary significant words.
\end{abstract}

\section{Introduction.}

De nombreux auteurs ont travaillé sur l'intelligibilité de la parole, bien que les matériels phonétiques utilisés soient différents et qu'ils appartiennent à des langues diverses. Les travaux de French et Steinberg en 1947, repris en 1962 par Kryter, ont permis de définir une méthode qui permet de calculer l'Indice d'Articulation (A.I.). Leurs travaux ont fortement contribué à l'établissement d'une norme par L'American National Standard Method en 1969 [1] donnant les directives du calcul de A.I. En effet, une fois calculé, cet indice permet de rendre compte de la qualité d'écoute ou d'évaluer le pourcentage d'intelligibilité pour des situations données. Fletcher et Galt [2] dont les travaux sont rejoints par Dirks en 1986 [3] proposaient un modèle de fonction de transfert :

$$
S=\left(1-10^{-\mathrm{A} / \mathrm{Q}}\right)^{\mathrm{N}}
$$

où $S$ représente les scores d'intelligibilité en fonction de l'indice d'articulation $A, Q$ et $N$ étant des constantes. Ce modèle nous a paru intéressant, bien que, pour notre étude, A ne puisse être lié directement à l'indice d'articulation. Néanmoins, son domaine de valeurs est respecté $(0<A<1)$ lorsque pendant les pas- 
sages de test d'intelligibilité, les conditions expérimentales nous limitaient à travailler en terme de rapport signal-sur-bruit dans une dynamique s'étalant entre $\mathrm{s} / \mathrm{b}=-40 \mathrm{~dB}$ à $\mathrm{s} / \mathrm{b}=+20 \mathrm{~dB}$. Ainsi, il est posé égal à :

$$
A=\frac{S / B+40}{60}
$$

D'autre part, pendant les études expérimentales où les mêmes diffuseurs acoustiques sont utilisés (un certain nombre) et où le type de local varie, le choix du modèle de l'équation (1) au nombre restreint de coefficients (avec A donné par (2)) semble dans un premier temps convenir. Ainsi notre travail consistera en partie à l'étude des paramètres $\mathrm{Q}$ et $\mathrm{N}$ qu'on associerait plutôt à l'enceinte et au local. La même méthodologie de passage de test est reportée en chambre sourde afin d'analyser l'influence des diffuseurs (de ses caractéristiques telles que directivité et spectre) et là où s'affranchit de celle du local.

\section{Procédures expérimentales.}

\subsection{Modes opératoires}

Des travaux préalables ont conduit à la mise au point d'une méthodologie de passage de test d'intelligibilité [4] où on utilise des listes de mots triphonémiques, phonétiquement équilibrées en accord avec les travaux de Haton et Lamotte conçernant le français parlé courant. Des sujets otologiquement sains ont pour mission de retranscrire les mots tels qu'ils les perçoivent. Il s'agit en fait d'une reconnaissance acoustique phonétique et non syntaxique. L'indice d'intelligibilité est déduit en calculant grâce à un logiciel de dépouillement mis au point pour la présente étude, le pourcentage de phonèmes correctement compris par le sujet. Le pourcentage d'intelligibilité moyen pour chaque groupe de sujets est calculé en faisant varier le rapport signal-sur-bruit relatif au signal de parole (dont le niveau continu équivalent est mesuré en $\mathrm{dB}$ (A)) [5] et à un bruit blanc masquant de $20 \mathrm{~Hz}-20 \mathrm{KHz}$.

\subsubsection{Expériences en salles réverbérantes.}

De telles procédures expérimentales étaient préalablement menées dans 3 locaux réverbérants de volumes respectifs $1000 \mathrm{~m}^{3}, 50000 \mathrm{~m}^{3}, 400000 \mathrm{~m}^{3}$ et dont les durées de réverbération TR1, TR2, TR3 moyennes mesurées par bande d'octave (de $63 \mathrm{~Hz}$ a $8 \mathrm{KHz}$ ) aux points de test (caractérisés par une certaine distance d par rapport au diffuseur (d: distance locuteur-auditeurs)) sont représentées dans le Tableau 1.

Tableau. 1. Durées de réverbération (s) moyennes pour les trois locaux de test.

\begin{tabular}{|c|c|c|c|c|c|c|c|c|}
\hline Local & 63 & 125 & 250 & 500 & 1000 & 2000 & 4000 & 8000 \\
\hline TR1 & 17.5 & 10 & 8.8 & 8.8 & 8.1 & 6.9 & 3.6 & 1.4 \\
\hline TR2 & 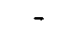 & 14.5 & 13.4 & 12 & 10 & 7.3 & 4.3 & 1.8 \\
\hline TR & - & 6.8 & 7.8 & 7.1 & 6.2 & 4.8 & 4.3 & - \\
\hline
\end{tabular}

Deux enceintes type Bouyer RB 90 et RB 33 sont destinées à la diffusion du message de parole. Une troisième enceinte type Bouyer RB 38 permet d'avoir le bruit de masque (dont le niveau est variable entre 60 et $80 \mathrm{dBA}$ ) délivré par un générateur B\&K type 1405. Contrairement à ce qu'a fait Santon qui étudiait dans ses récents travaux [6] le Seuil de Réception de la Parole (SRP) pour différentes directions du bruit de masque, ce dernier est dans notre cas diffusé face aux auditeurs $\left(\varnothing=0^{\circ}\right)$ pendant tous les passages de test.

\subsubsection{Expériences en chambre sourde.}

Les mêmes expérimentations sont reportées en chambre sourde avec les mêmes matériels de test (mêmes enceintes et mêmes listes de mots) afin de faire une analyse plus fine des coefficients de la régression (équation (1)). Une seule distance $\mathrm{d}=4 \mathrm{~m}$ est prise pour éviter toute perturbation de champ proche. Deux directions $0^{\circ}$ et $90^{\circ}$ sont choisies pour diffuser le signal de parole quand la RB90 est utilisée. Des corrections spectrales en bruit blanc (filtré par 1/3 d'octave) sont apportées (à l'aide d'égaliseurs de marque ALTEC placée dans la chaîne d'émission) de façon à ce que l'on obtienne le spectre émis de face quand l'enceinte est placée de profil et vice versa. On obtient ainsi 5 configurations d'enceintes (Tableau.3) pour l'émission des listes de mots. On étudiera de cette façon l'effet de la directivité autre que celui dû au changement de spectre.

\section{Etudes du modèle et Résultats}

Le modèle choisi permet de retrouver la caractéristique en " $\mathrm{S}$ " des courbes d'intelligibilité rencontrées courament dans la littérature[2,3] quand elles sont tracées en fonction de l'indice d'articulation. En effet, lors de nos expérimentations où $\mathrm{A}$ est donné par (2), il se trouve que la dynamique de travail devrait s'étaler entre 
$\mathrm{S} / \mathrm{B}=-20 \mathrm{dBA}$ et $\mathrm{S} / \mathrm{B}=+20 \mathrm{dBA}$ dans les locaux réverbérants $T R 1$, TR2, TR3 et est décalée vers la gauche entre les valeurs $S / B=-40 \mathrm{dBA}$ et $\mathrm{S} / \mathrm{B}=0 \mathrm{dBA}$ en chambre sourde. On souligne par cette constatation l'effet de la réverbération qui dégrade l'intelligibilité[7]. Dans tous les cas, un calcul de minimisation de l'erreur quadratique $\Delta E$ entre le modèle de régression et les scores expérimentaux est ensuite effectué pour avoir les coefficients $\mathrm{Q}$ et $\mathrm{N}$ dont les valeurs figurent dans les Tableau. 2 et 3 .

Tableau.2. Valeurs des coefficients pour les 3 locaux testés. F:Enceinte utilisée de Face;

P:Enceinte utilisée de Profil.

\begin{tabular}{|c|c|c|c|c|c|c|c|c|c|}
\hline Locaux & \multicolumn{3}{|c|}{$\begin{array}{l}\text { Salle réverbérante } \\
\text { Intespace(Toulouse) }\end{array}$} & \multicolumn{3}{|c|}{$\begin{array}{c}\text { Eglise des } \\
\text { Augustins(Toulouse) }\end{array}$} & \multicolumn{3}{|c|}{$\begin{array}{c}\text { Basilique } \\
\text { Yamoussoukro }\end{array}$} \\
\hline Diffuseur & dist. & $\bar{Q}$ & $\bar{N}$ & dist. & $\bar{Q}$ & $\bar{N}$ & dist. & $Q$ & $\mathrm{~N}$ \\
\hline \multirow{3}{*}{ RB 33} & $2 m$ & 0.27 & $\overline{72.82}$ & $2 m$ & 0.22 & 88.78 & $10 \mathrm{~m}$ & 0.42 & 13.04 \\
\hline & $4 \mathrm{~m}$ & 0.30 & 70.13 & $8 \mathrm{~m}$ & 0.26 & 107.1 & $20 \mathrm{~m}$ & 0.42 & 30.02 \\
\hline & $6 \mathrm{~m}$ & 0.31 & 95.90 & $16 \mathrm{~m}$ & 0.40 & 31.98 & $40 \mathrm{~m}$ & 0.32 & 140 \\
\hline \multirow{3}{*}{ RB90 F } & $2 \mathrm{~m}$ & 0.29 & 39.60 & $2 m$ & 0.20 & 111.9 & $10 \mathrm{~m}$ & 0.39 & 26.87 \\
\hline & $4 \mathrm{~m}$ & 0.27 & 36.24 & $8 \mathrm{~m}$ & 0.24 & 66.36 & $20 \mathrm{~m}$ & 0.24 & 367.4 \\
\hline & $6 \mathrm{~m}$ & 0.27 & 52.26 & $16 \mathrm{~m}$ & 0.32 & 30.68 & $40 \mathrm{~m}$ & 0.57 & 15.72 \\
\hline \multirow{3}{*}{ RB90 P } & $2 \mathrm{~m}$ & 0.36 & 18.94 & $2 m$ & 0.47 & 5.31 & $10 \mathrm{~m}$ & 0.61 & 4.84 \\
\hline & $4 \mathrm{~m}$ & 0.35 & 23.57 & $8 m$ & 0.45 & 20.95 & $20 \mathrm{~m}$ & 0.70 & 7.49 \\
\hline & $6 \mathrm{~m}$ & 0.45 & 14.97 & $16 \mathrm{~m}$ & 0.77 & 6.94 & $40 \mathrm{~m}$ & 1.88 & 2.60 \\
\hline
\end{tabular}

Tableau.3. Scores d'Intelligibilité ( en \%) en chambre sourde obtenus à d=4m.

\begin{tabular}{|c|c|c|c|c|c|c|c|c|}
\hline \multirow{2}{*}{$\begin{array}{c}\text { S/B } \\
\text { Enceinte }\end{array}$} & -17.5 & -15 & -12.5 & -10 & -7.5 & -5 & \multirow{2}{*}{\multicolumn{2}{|c|}{$\mathrm{Q} \quad \mathrm{N}$}} \\
\hline & & & & & & & & \\
\hline $\begin{array}{l}\text { RB33 } \\
\text { RB90 }\end{array}$ & & 41 & 53 & 69 & 74 & 78 & 0.27 & 29 \\
\hline $\begin{array}{l}\text { RB90 } \\
\text { RB90 }\end{array}$ & 26 & 45 & $\begin{array}{l}52 \\
56\end{array}$ & $\begin{array}{l}69 \\
66\end{array}$ & 79 & 82 & $\begin{array}{l}0.24 \\
0.40\end{array}$ & $\begin{array}{c}46.74 \\
7.31\end{array}$ \\
\hline $\begin{array}{l}\text { RB90 } \\
\text { RB90 }\end{array}$ & $\begin{array}{l}43 \\
46\end{array}$ & $\begin{array}{l}48 \\
50\end{array}$ & $\begin{array}{l}56 \\
59\end{array}$ & $\begin{array}{l}00 \\
63\end{array}$ & 70 & - & $\begin{array}{l}0.40 \\
0.54\end{array}$ & 3.49 \\
\hline $\mathrm{RB}$ & 50 & 65 & 71 & 69 & 82 & & 0.38 & 5.8 \\
\hline
\end{tabular}

FC: Enceinte travaillant de Face mais dont le spectre est Corrigé pour être celui de l'enceinte de profil ;

PC: Enceinte travaillant de Profil mais dont le spectre est Corrigé pour être celui de l'enceinte de face.

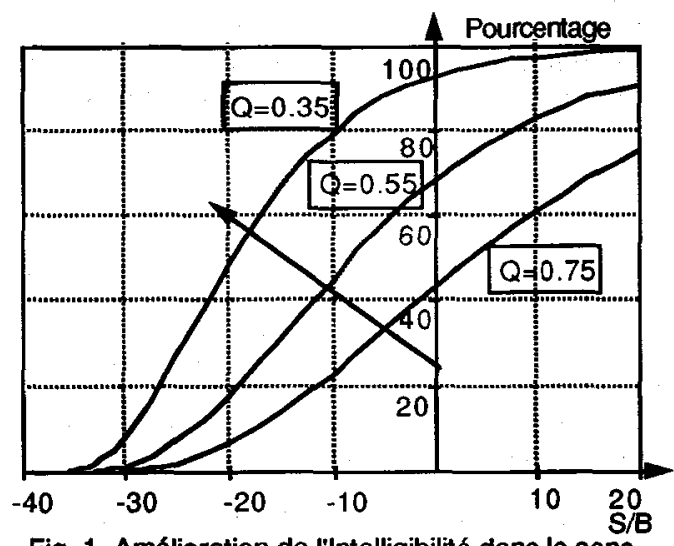

Fig. 1. Amélioration de I'Intelligibilité dans le sens des $Q$ décroissants.( $N$ fixé).

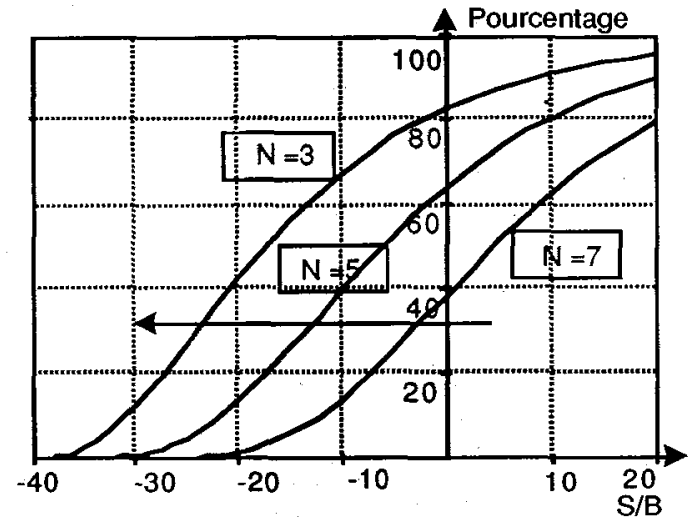

Fig. 2. Amélioration de I'Intelligibilité dans le sens des $\mathbf{N}$ décroissants ( $Q$ fixé).

3.1. Coefficient $Q$ : Les corrections spectrales apportées permettent de classer les diffuseurs comme des configurations "de face" ou "de profil" (cas de la RB90 en chambre sourde). Par la suite, d'une manière générale, on remarque que quel que soit le type de local, l'enceinte émettant de face correspond à une valeur du coefficient Qmoyen inférieure à ce qu'elle est lorsque l'enceinte travaille de profil (Tableau.2 et Tableau.3). Sa valeur semble augmenter quand la distance locuteur-auditeur croît. Cette constatation est surtout remarquable dans les locaux de volume moyen et où la durée de réverbération évolue de façon exponentielle par bande d'octave (cas du local TR2). De plus sa valeur présente relativement peu de 
fluctuations. La première remarque permettrait de confirmer l'hypothèse posée telle que le coefficient $Q$ puisse dépendre directement de l'enceinte (de sa directivité ou de l'effet de son spectre). D'autre part, une étude qualitative de variation du paramètre $Q$ pour une valeur de $N$ constante entraîne une rotation (autour de $S / B=-30 \mathrm{dBA}$ ) de la courbe (Figure 1) vers les rapports S/B défavorables. Des petites valeurs du coefficient $\mathrm{Q}$ améliorent le score d'intelligibilité pour une même valeur du rapport $\mathrm{S} / \mathrm{B}$.

3.1. Coefficient $N$ : Contrairement au paramètre $\mathrm{Q}$, le coefficient $\mathrm{N}$ ne semble pas vérifier l' hypothèse posée au départ telle qu'il dépende plutôt de la salle. En effet, sa valeur ne reste pas constante pour un local donné par exemple en chambre sourde, comme on aurait pu le penser. Elle présente des fluctuations importantes comparativement à celles du coefficient $Q$. On peut noter par contre une variation moyenne de $\mathrm{N}$ en sens inverse de $\mathrm{Q}$. Il prend une valeur supérieure quand l'enceinte diffuse de face. Ce paramètre $\mathrm{N}$ caractérise une translation de la courbe vers les rapports $\mathrm{S} / \mathrm{B}$ favorables pour des valeurs de $\mathrm{N}$ croissantes a $\mathrm{Q}$ constant (Figure 2). Une plus faible valeur de $\mathrm{N}$ conserve donc le même pourcentage d'intelligibilité quand le rapport Signal-sur-Bruit se dégrade.

4.Commentaires et Conclusions: L'expérimentation que nous avons menée permet de souligner les résultats suivants :

-L'intelligibilité croît en 1/d (d: distance locuteur- auditeur),

-Cependant, dans certains cas, cette règle est inversée (cf. Tableau. 2 RB $90 \mathrm{~F}$ à $4 \mathrm{~m}$ par rapport à $2 \mathrm{~m}$ ). Cette enceinte est multivoies, de grandes dimensions; de ce fait, le tweeter n'est pas dans l'axe de l'auditeur et le spectre à faible distance est différent de celui reçu à une distance optimale.

-Il est connu que, l'intelligibilité croît quand le rapport Signal-sur-Bruit augmente [8]. Dans notre cas, on a constaté que cela reste valable quel que soit le type de local et le mode de diffusion de l'enceinte (en spectre corrigé ou non, de face comme de profil ).

-En situation de champ direct, et pour des rapports Signal-sur-Bruit inférieurs à $S / B=-10 \mathrm{dBA}$, une émission à $90^{\circ}$ du signal de parole (bruit de masque dans l'axe et spectre de parole corrigé), améliore de façon notable la compréhension du message (cf Tableau 3 entre RB90 F et RB90 PC). Ce résultat serait représentatif d'un phénomène de démasquage dans la direction perpendiculaire $\left(\varnothing=90^{\circ}\right)$. Il pourrait s'expliquer d'autre part par un pouvoir de discrimination spatiale des sujets "otologiquement sains" qui peuvent faire abstraction dans une certaine mesure des informations nuisibles provenant de la direction frontale [6].

-Dans le cas de diffusion frontale, avec correction de spectre, celle-ci conduisant à une atténuation par bande $1 / 3$ d'octave de $17 \mathrm{~dB}$ en moyenne entre $630 \mathrm{~Hz}$ et $10 \mathrm{KHz}$, l'intelligibilité n'est pas affectée.

-A une valeur particulière du rapport Signal-sur-Bruit (autour de $S / B=-10 \mathrm{~dB}$ ), toutes les enceintes travaillant de face fournissent le même score d'intelligibilité. Au delà de cette valeur $(\mathrm{S} / \mathrm{B}>-10 \mathrm{~dB})$, la directivité de l'enceinte semble être traduite par l'effet de son spectre. Dans cette zone de rapports S/B, les scores d'intelligibilité sont les mêmes à spectre égal, que l'enceinte soit de face ou de profil.

Enfin, il convient de noter que la méthodologie utilisée conduit à une erreur maximale sur les scores inférieure à $\Delta \mathrm{I}=10 \%$ (prise en compte des erreurs sur les mesures des rapports Signal-sur-Bruit et le calcul des coefficients).

\section{Bibliographie}

[1] American National Standards Institute.:"American national standard methods for the calculation of the articulation index".A.N.S.I, S3.5- New-York (1969).

[2] Fletcher H., and Galt R.H. :"The perception of speech and its relation to telephony" J. Acoust. Soc . Am. 22, (1950), 89-151.

[3] Dirks D. D. et Al::"Articulation index predictors of contextually dependent words".J. Acoust. Soc . Am. 80 (1), (1986), 82-92.

[4] Legros C., Gamba R., Zuliani.P. :"Intelligibilité de la parole dans le bruit: Elaboration d'une méthode de diagnostic applicable in-situ en présence de bruit fluctuant". Contrat Ministère de l'environnement $n^{\circ} 85$ $333,(1988)$, Compte rendu final.

[5] Zuliani.P. :"Intelligibilité de la parole dans des conditions d'écoute difficiles: étude et prévision fondées sur l'hypothèse de réflexion diffuse en parois".Thèse de 3è cycle, Toulouse III, (1988).

[6] Santon F. :"Intelligibilité de la parole masquée et rôle de la direction de la source masquante" Acustica Vol. 61, (1986), 67-74.

[7] Legros C., Gamba R., Zuliani.P.:"Quelques paramètres influant sur l'intelligibilité de la parole dans des conditions d'ecoute difficiles". $8^{\mathrm{e}}$ Symposium FASE sur l'Acoustique de l'environnement, Proceedings, 24-28 Avril, (1989), 169-172.

[8] Bradley J. S. :"Predictors of speech intelligibility in rooms" J. Acoust. Soc. Am. 80 (3), (1986), $837-845$. 